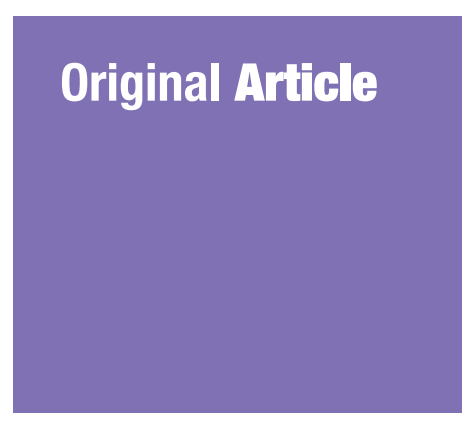

Submitted: 13 Oct 2020

Accepted: 23 Feb 2021

Online: 30 Jun 2021

\section{Parcellation of the Hippocampus According to Its Connection Probability with Prefrontal Cortex Subdivisions in a Malaysian Malay Population: Preliminary Findings}

\author{
Aimi Nadhiah Abdullah ${ }^{1}$, Asma Hayati Ahmad", \\ Rahimah ZAKARIA ${ }^{1}$, Sofina TAMAM${ }^{2}$, Jafri Malin ABduLlaH ${ }^{3,4,5}$ \\ 1 Department of Physiology, School of Medical Sciences, \\ Universiti Sains Malaysia, Kubang Kerian, Kelantan, Malaysia \\ 2 Faculty of Science and Technology, Universiti Sains Islam Malaysia, \\ Negeri Sembilan, Malaysia \\ 3 Department of Neurosciences, School of Medical Sciences, \\ Universiti Sains Malaysia, Kubang Kerian, Kelantan, Malaysia \\ 4 Brain and Behaviour Cluster, School of Medical Sciences, \\ Universiti Sains Malaysia, Kubang Kerian, Kelantan, Malaysia \\ 5 Hospital Universiti Sains Malaysia, Kubang Kerian, Kelantan, Malaysia
}

To cite this article: Abdullah AN, Ahmad AH, Zakaria R, Tamam S, Abdullah JM. Parcellation of the hippocampus according to its connection probability with prefrontal cortex subdivisions in a Malaysian Malay population: preliminary findings. Malays J Med Sci. 2021;28(3):65-76. https://doi.org/10.21315/mjms2021.28.3.6

To link to this article: https://doi.org/10.21315/mjms2021.28.3.6

\begin{abstract}
Background: Lesion studies have shown distinct roles for the hippocampus, with the dorsal subregion being involved in processing of spatial information and memory, and the ventral aspect coding for emotion and motivational behaviour. However, its structural connectivity with the subdivisions of the prefrontal cortex (PFC), the executive area of the brain that also has various distinct functions, has not been fully explored, especially in the Malaysian population.

Methods: We performed diffusion magnetic resonance imaging with probabilistic tractography on four Malay males to parcellate the hippocampus according to its relative connection probability to the six subdivisions of the PFC.

Results: Our findings revealed that each hippocampus showed putative connectivity to all the subdivisions of PFC, with the highest connectivity to the orbitofrontal cortex (OFC). Parcellation of the hippocampus according to its connection probability to the six PFC subdivisions showed variability in the pattern of the connection distribution and no clear distinction between the hippocampal subregions.

Conclusion: Hippocampus displayed highest connectivity to the OFC as compared to other PFC subdivisions. We did not find a unifying pattern of distribution based on the connectivitybased parcellation of the hippocampus.
\end{abstract}

Keywords: diffusion magnetic resonance imaging, hippocampus, prefrontal cortex 


\section{Introduction}

The hippocampus, a medial temporal lobe structure found in all species of mammals, plays a key role in spatial navigation as well as in various modes of learning and memory (13 ). The entorhinal cortex, which connects the hippocampus with the rest of the neocortex, is a main source of its inputs (4). The hippocampus is divided into many subfields including the regions of dentate gyrus and cornum ammonis areas 1 and 3 . It is also arranged along a longitudinal axis that stretches from an anterior to a posterior pole in primates, as well as in humans (5). Lesion studies have shown that tasks usually associated with the hippocampus (i.e. the processing of spatial information and memory) are mostly subserved by its dorsal subregion, while the ventral hippocampus is more involved in emotional and motivational behaviours such as anxiety (6). The unique anatomical connections of the dorsal and ventral poles to both afferent and efferent structures also reflect this functional dissociation $(7,8)$.

The prefrontal cortex (PFC) is a more phylogenetically divergent structure than the hippocampus (4), and it is a critical area for cognitive processes and emotional control of the higher order. The primate PFC is structured into many subregions but can be primarily divided into a dorsolateral division involving cognitive functions such as executive control, attention and working memory, and a ventromedial (or orbitomedial) division more involved in emotional and motivational regulation $(9,10)$. The PFC receives monosynaptic projections from the hippocampus in both rodents and primates (11-13). These projections originate almost exclusively in the ventral hippocampus and primarily target the medial PFC (mPFC), with some evidence suggesting stronger projections to ventral subregions (11-14). In addition to these monosynaptic connections, bidirectional interactions between the two structures also occur via many indirect routes. One potential relay is the thalamus nucleus reuniens, which is connected reciprocally to both the dorsal and ventral hippocampus as well as the mPFC (15, 16).

Various approaches can be used to study hippocampal-prefrontal interactions in animals and humans. One of them is using diffusion magnetic resonance imaging (dMRI), a method that makes exploration of white matter connectivity in the living brain feasible. The development of dMRI has enabled the evaluation of white matter tracts by virtue of its ability to image water diffusion characteristics (17). In early dMRI studies, deterministic tractography, which estimates only the primary orientation of diffusion in each magnetic resonance imaging (MRI) voxel, was commonly used $(17,18)$. However, due to crossing fibres, this method was unable to trace all the connected brain regions from the seed voxels $(17,18)$. In contrast, probabilistic tractography, which is a reflection of the multiple orientations of diffusion and an estimation of more than one fibre population in each MRI voxel, enables tracing of the crossing fibres (17-19).

Accordingly, probabilistic tractography has been widely used for the investigation of the neural connectivity of neural structures in the human brain, including the fornix, lateral geniculate body and red nucleus, to name a few (20-23). To date, a few dMRI studies are available on the anatomical neural tracts between the hippocampus and prefrontal regions (24). However, the possible variability in the structural connectivity between the hippocampus and the six prefrontal subdivisions has yet to be fully explored. In the present study, using probabilistic dMRI tractography, we attempted to track the hippocampal-prefrontal cortex connectivity in healthy Malaysian subjects.

\section{Methods}

\section{Subjects}

Four healthy male subjects with no previous history of neurological, physical or psychiatric illness were recruited for this study. These subjects were healthy controls of another related study involving traumatic brain injury patients. All subjects were right handed, understood the purpose of the study and provided written, informed consent prior to participation. Magnet safety screening was performed prior to scanning.

\section{Data Acquisition}

MRI scan images were acquired using a $3 \mathrm{~T}$ Philips Achieva MRI scanner (Netherlands) with a 32-channel SENSE head coil. The protocol for the dMRI was as follows: repetition time $(\mathrm{TR})=10,726 \mathrm{~ms}$, echo time $(\mathrm{ET})=76 \mathrm{~ms}$, field of view $($ FOV $)=221 \times 221$, matrix $=96 \mathrm{~mm}$ $\times 94 \mathrm{~mm}$, slice thickness $=2.3 \mathrm{~mm}, 67$ slices, $b=1,000 \mathrm{~s} / \mathrm{mm}^{2}$, voxel size of $2.3 \times 2.3 \times$ 
$2.3 \mathrm{~mm}^{3}$, and EPI factor $=57$ resulting in 32 diffusion weighted volumes $\left(b=1,000 \mathrm{~s} / \mathrm{mm}^{2}\right)$ and one non-diffusion weighted volume $\left(b=0 \mathrm{~s} / \mathrm{mm}^{2}\right)$ as reference. T1-weighted image was acquired using the following parameters: $\mathrm{TR}=7.4 \mathrm{~ms}, \mathrm{TE}=3.4 \mathrm{~ms}, \mathrm{FOV}=250 \times 250$, matrix size $=228 \mathrm{~mm} \times 227 \mathrm{~mm}$, voxel size $=$ $1.1 \mathrm{~mm} \times 1.1 \mathrm{~mm}$, slice thickness $=1.2 \mathrm{~mm}$ and 240 slices. Acquisition of the diffusion imaging data took 7 min per subject.

\section{Pre-Processing}

Data preprocessing utilised tools from FDT (FMRIB's Diffusion Toolbox), part of FMRIB's Software Library (FSL version 5.0.9). Diffusionweighted images were initially corrected for head motion effect and image distortion due to eddy currents. Probability density functions on up to two principal fibre directions were estimated at each voxel in the brain using the Bayesian
Estimation of Diffusion Parameters obtained using sampling techniques toolbox (bedpostx; 17) implemented in FSL.

\section{Definition of Regions of Interest in Structural Space}

Regions of interest masks were bilaterally hand-drawn on the T1-weighted image (structural space) for each subject according to the anatomical landmarks referred to in the Duvernoy's atlas of the human brain (25) as shown in Figure 1. The regions of interest included hippocampus as the seed and the six subdivisions of the PFC (Table 1) as the targets, namely the dorsolateral prefrontal cortex (DLPFC), ventrolateral prefrontal cortex (VLPFC), frontopolar cortex (FPC), orbitofrontal cortex (OFC), ventromedial prefrontal cortex (VMPFC) and dorsomedial prefrontal cortex (DMPFC) $(24,26,27)$.
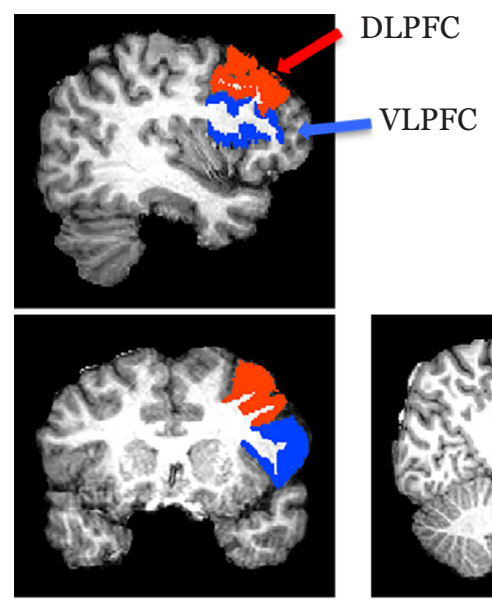

DMPFC

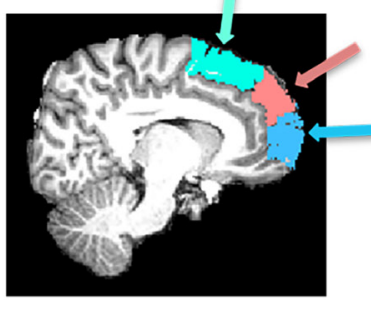

\section{VMPFC}

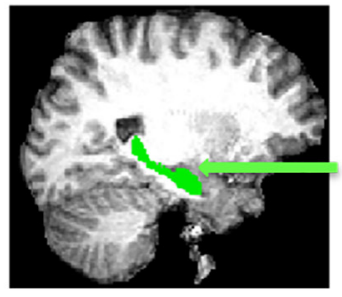

Hippocampus

Frontopolar

Orbitofrontal cortex

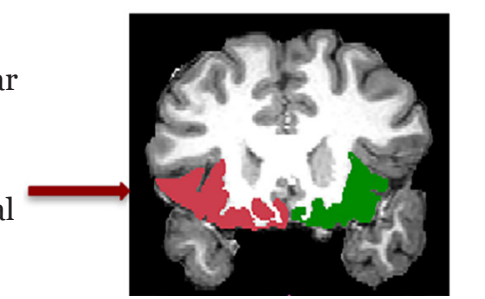

Figure 1. Regions of interest hand-drawn in the structural space of one subject. Seed mask: hippocampus; target masks: six subdivisions of the prefrontal cortex i.e., DLPFC, VLPFC, FPC, OFC, VMPFC and DMPFC

Table 1. Subdivisions of PFC according to Brodmann areas (BA)

\begin{tabular}{cll} 
No & PFC subdivisions & BA \\
\hline 1 & DLPFC & BA 8, 9, 46 and 9/46 in the superior and middle IFG (28) \\
2 & VLPFC & $\begin{array}{l}\text { BA 44 (pars opercularis), 45 (pars triangularis) and lateral part of area } \\
\text { 47/12 of IFG }(28,29)\end{array}$ \\
& & BA 10 (30) \\
3 & FPC & BA 10, 11, 12, 13, 14 and orbital part of area 47/12 (29, 31) \\
4 & OFC & BA 10, 14, subgenual cingulate cortex (BA 32 and 25) and ventral ACC (32) \\
5 & VMPFC & Medial regions of BA 8, 9 and 9/46 (30) \\
6 & DMPFC &
\end{tabular}




\section{Probabilistic Tractography}

Fibre tracking was performed using probtrackx module implemented in FSL, following the method previously described by Behrens et al. (28). The probability distribution of the principal diffusion direction was estimated at each voxel, and the estimated distribution represented uncertainty in the diffusion direction caused by factors that include potential co-existence of many fibre pathways within a single voxel, image noise and subject movement in the scanner (29). The algorithm of probabilistic tractography utilises these local probability distributions to generate streamline samples (fibre pathways) to build up the connectivity distribution in the structural space. Probabilistic tractography was performed using a single-seed approach. The tracking parameters included 5,000 samples per voxel, a step length of $0.5 \mathrm{~mm}$, and a curvature threshold of 0.2. Probabilistic tractography maps were individually generated for the left and right halves of the masks.

\section{Calculation of Relative Connection Probability}

Connection probability, which indicates the probability that a sample initiated from a seed region will reach a particular target region, was obtained from the output of probtrackx. From the 5,000 samples initiated from each voxel in the seed region, the number of samples that reaches the target was multiplied by the number of voxels in the seed area with positive connection probability to the target. The relative connection probability is the percentage of the connection probability for each target over the sum of the connection probabilities to all targets $(27,30)$.

\section{Statistical Analysis}

Repeated measures ANOVA using SPSS software (v.24.o; SPSS, Chicago, IL) was only used to determine whether there was any differences in the volumes of seed and target masks between the left and right hemispheres. Statistical significance was accepted for $P$-values of less than 0.05.

\section{Results}

\section{Demographic Data}

All subjects were male, age ranged between 21 and 52 years old (mean [SD] = 30.6 [14.4]), right hand dominant, possessed at least nine years of education, had no psychiatric illness and not on any psychiatric drugs (Table 2).

Table 2. Participants' particulars

\begin{tabular}{ccc} 
Participant & $\begin{array}{c}\text { Age } \\
\text { (years old) }\end{array}$ & $\begin{array}{c}\text { Education } \\
\text { (years) }\end{array}$ \\
1 & 26 & 17 \\
2 & 52 & 11 \\
3 & 21 & 14 \\
4 & 23 & 16 \\
\hline
\end{tabular}

\section{Region of Interest Mask Sizes}

The comparison of the mask sizes was done to ensure the high accuracy of the mask and uniformity of the mask sizes since the masks were hand-drawn. The different sizes of the seed and target masks are summarised in Figure 2. The biggest mask was the one for the DLPFC (left side, mean $[\mathrm{SD}]=28,307.00[3,626.56]$ ); right side, mean $[\mathrm{SD}]=29,659.00[5,482.86])$ and the smallest was the one for hippocampus (left side, mean $[\mathrm{SD}]=5,850.50$ [668.76]; right side, mean $[\mathrm{SD}]=5,815.00$ [558.53]). Repeated measures ANOVA with two factors, hemisphere (left and right) and PFC subdivisions (DLPFC, VLPFC, FPC, OFC, VMPFC and DMPFC) showed that the prefrontal masks were not significantly different between hemispheres (main effects of hemisphere: $F(5,30)=0.35, P=0.88$ ) but there was a significant main effect of PFC subdivisions: $F(5,30)=36.18, P<0.001)$ meaning that the mask sizes for both hemispheres were not significantly different and the differences among the different subregions were as expected.

\section{Tractography Results}

Tractography was used to compare the relative connection probability between hippocampus and the six subdivisions of the PFC. Individual tracking of the hippocampalprefrontal tractography revealed variable patterns of connectivity among the subjects and between the right and left hemispheres. In general, the hippocampus showed connectivity to all of the subdivisions of the PFC (i.e., the DLPFC, VLPFC, OFC, FPC, VMPFC and 
Original Article | Parcellation of hippocampus

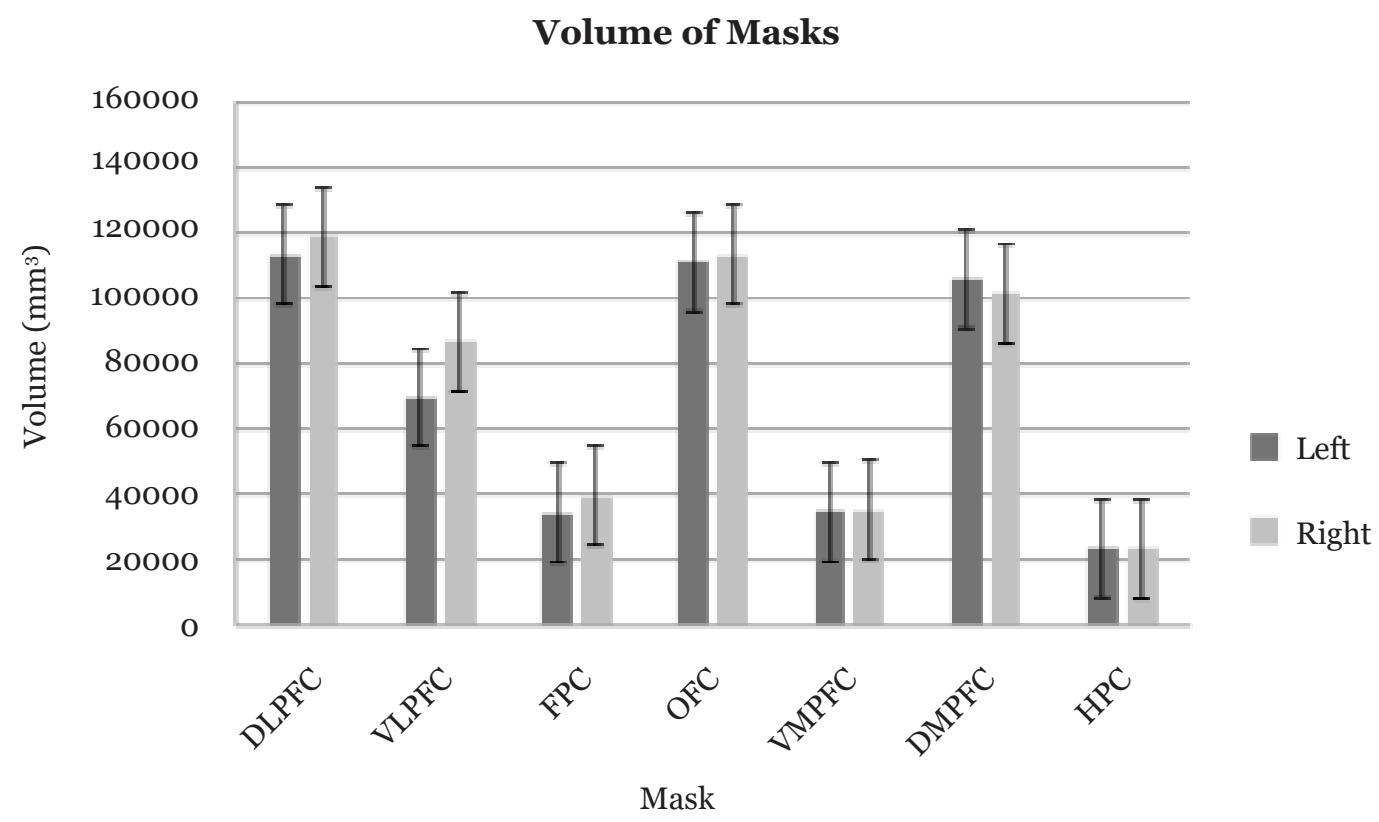

Figure 2. Volumes of seed (hippocampus) and target masks (PFC subdivisions DLPFC), VLPFC, FPC, OFC, VMPFC and DMPFC. Values are mean \pm SEM

\section{Subject 1}
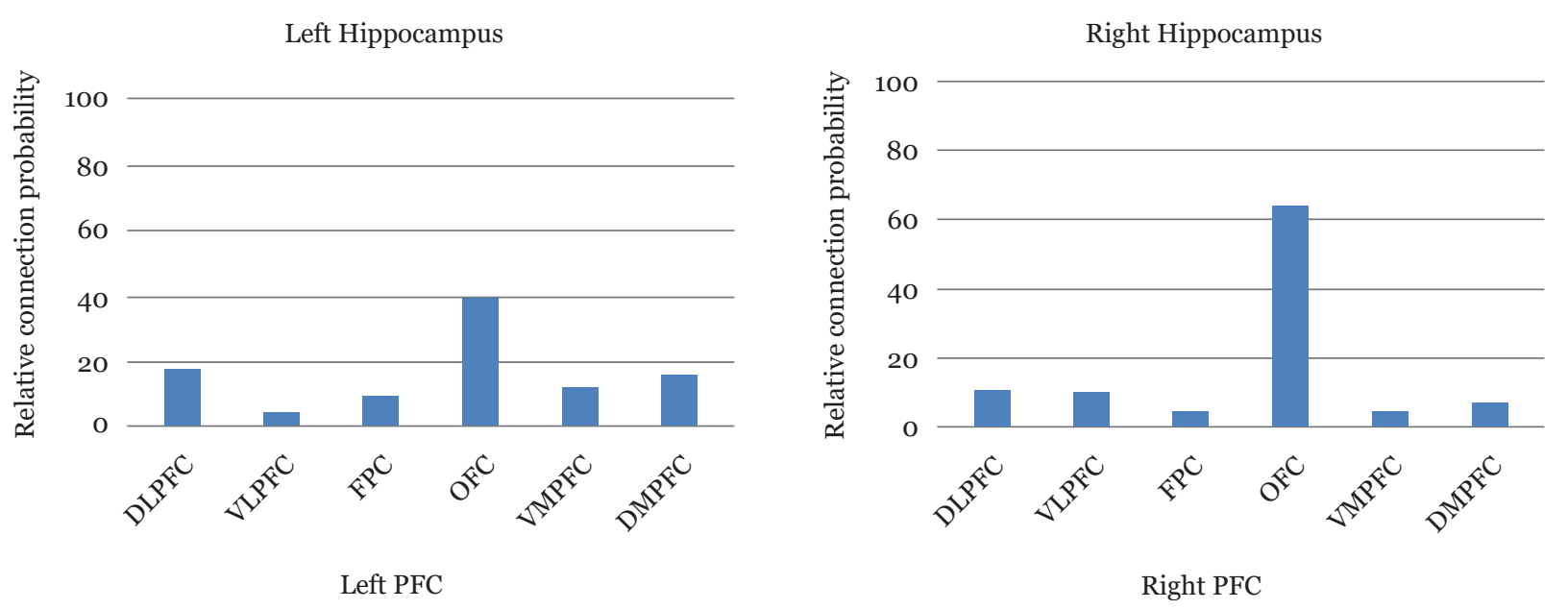

(continued on next page)

Figure 3. Relative connection probability from hippocampus to six PFC subdivisions on the left and right hemisphere for each subject $(n=4)$ 


\section{Subject 2}

Left Hippocampus

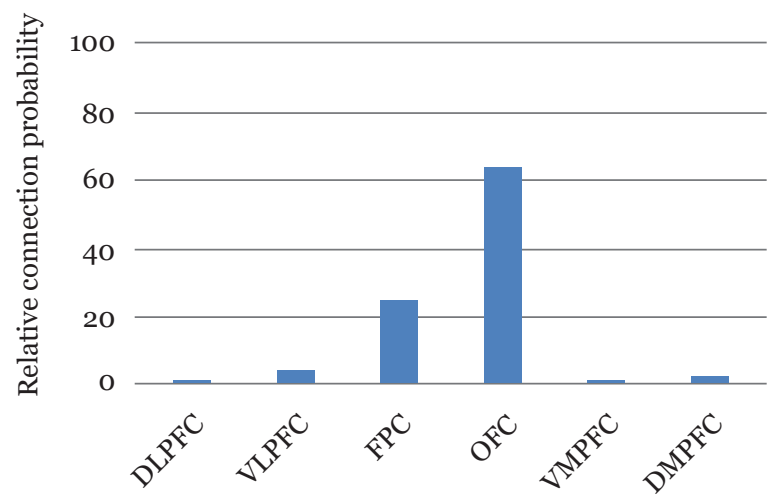

Left PFC

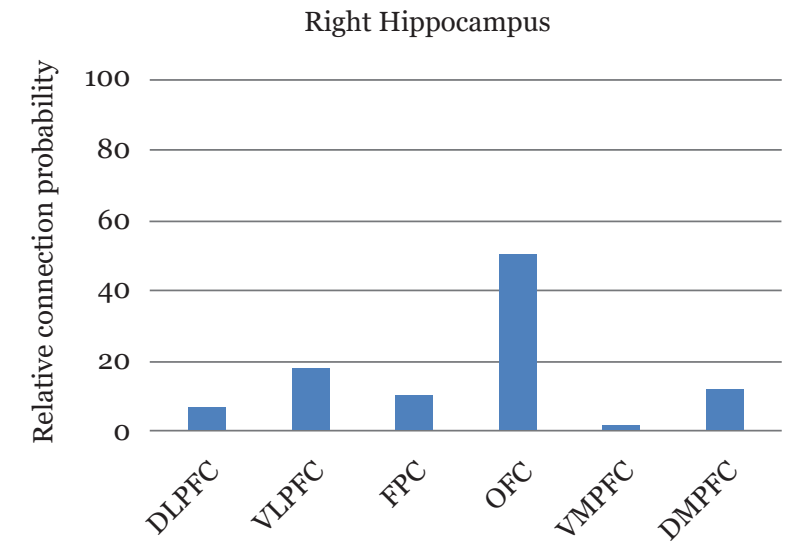

Right PFC

\section{Subject 4}

Left Hippocampus

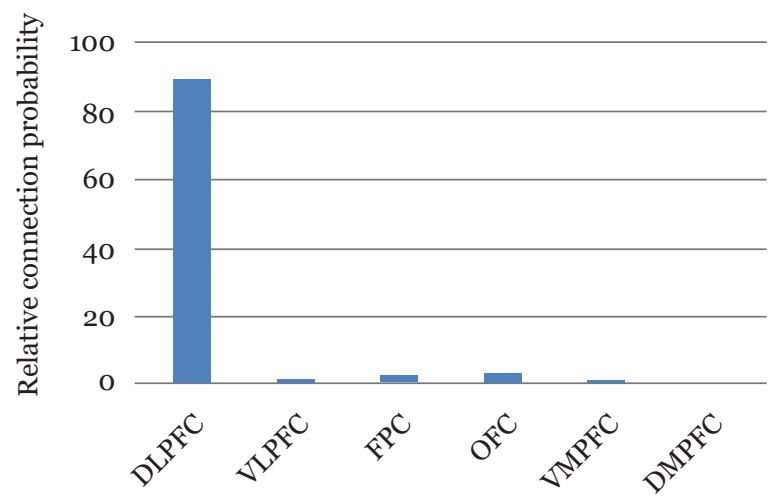

Left PFC

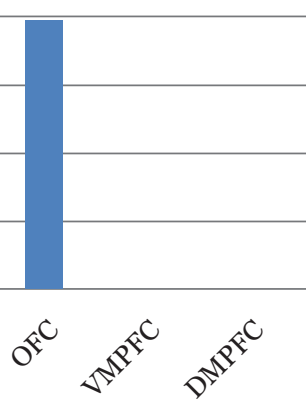

Left PFC
Right Hippocampus

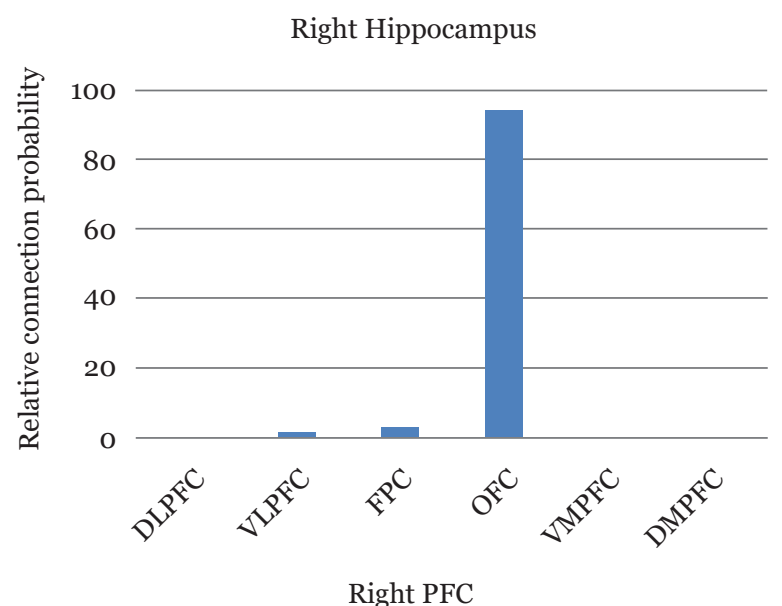

Right PFC

Right Hippocampus

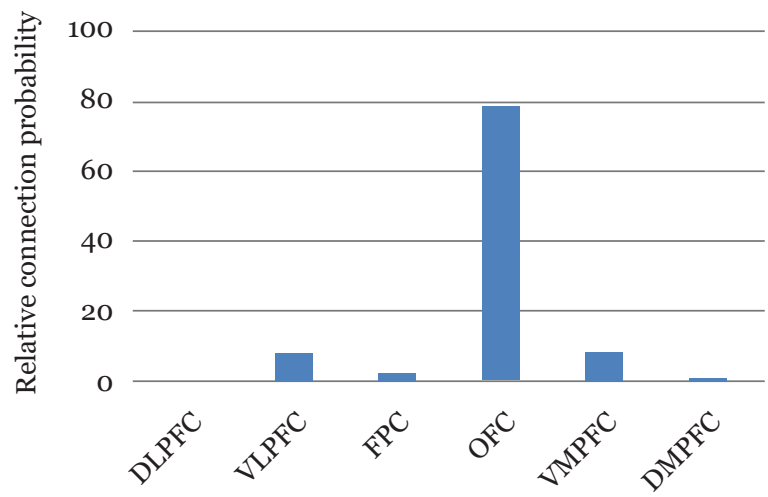

Right PFC

Figure 3. (continued) 
Original Article | Parcellation of hippocampus

DMPFC). However, there was a high degree of variability among subjects in terms of the pattern of distribution. In general, the highest connectivity was to the OFC in all subjects for both hemispheres (Figure 3).

Subject 1

The highest connection probability was to the OFC for both the left and right hemispheres, with the right side displaying higher connection probability as compared with the other PFC subdivisions. Connection probability to the other subdivisions of the PFC was less than $20 \%$ each.

Subject 2

Similar to subject 1 , the highest connection probability was to the OFC. The left hippocampus displayed a relatively lower percentage of connection probability to the other PFC subdivisions except for the FPC.

\section{Subject 3}

Connection probabilities for both hemispheres were predominantly to $\mathrm{OFC}$, while those to all the other PFC subdivisions were much lower except to the VLPFC over the left hemisphere only.
Subject 4

Different from the other subjects, the highest connectivity over the left hemisphere was to the DLPFC with much lower connection probability to all the other PFC subdivisions. Over the right hemisphere, however, the pattern was quite similar to the other subjects with the highest connection probability to the OFC, while the connection probabilities to the other PFC subdivisions were less than $20 \%$.

\section{Parcellation}

Parcellation of the hippocampus can indicate where the hippocampus has the highest connection probability with each $\mathrm{PFC}$ subdivision. The results showed variable patterns of distribution among the four subjects (Figures 4 and 5). The OFC was visually prominent in most subjects' hippocampus, either in the medial or lateral view. Another PFC subdivision that featured prominently was the DLPFC, especially in subjects 3 and 4 . However, the parcellation maps displayed no distinct pattern that would indicate segregation between the dorsal and

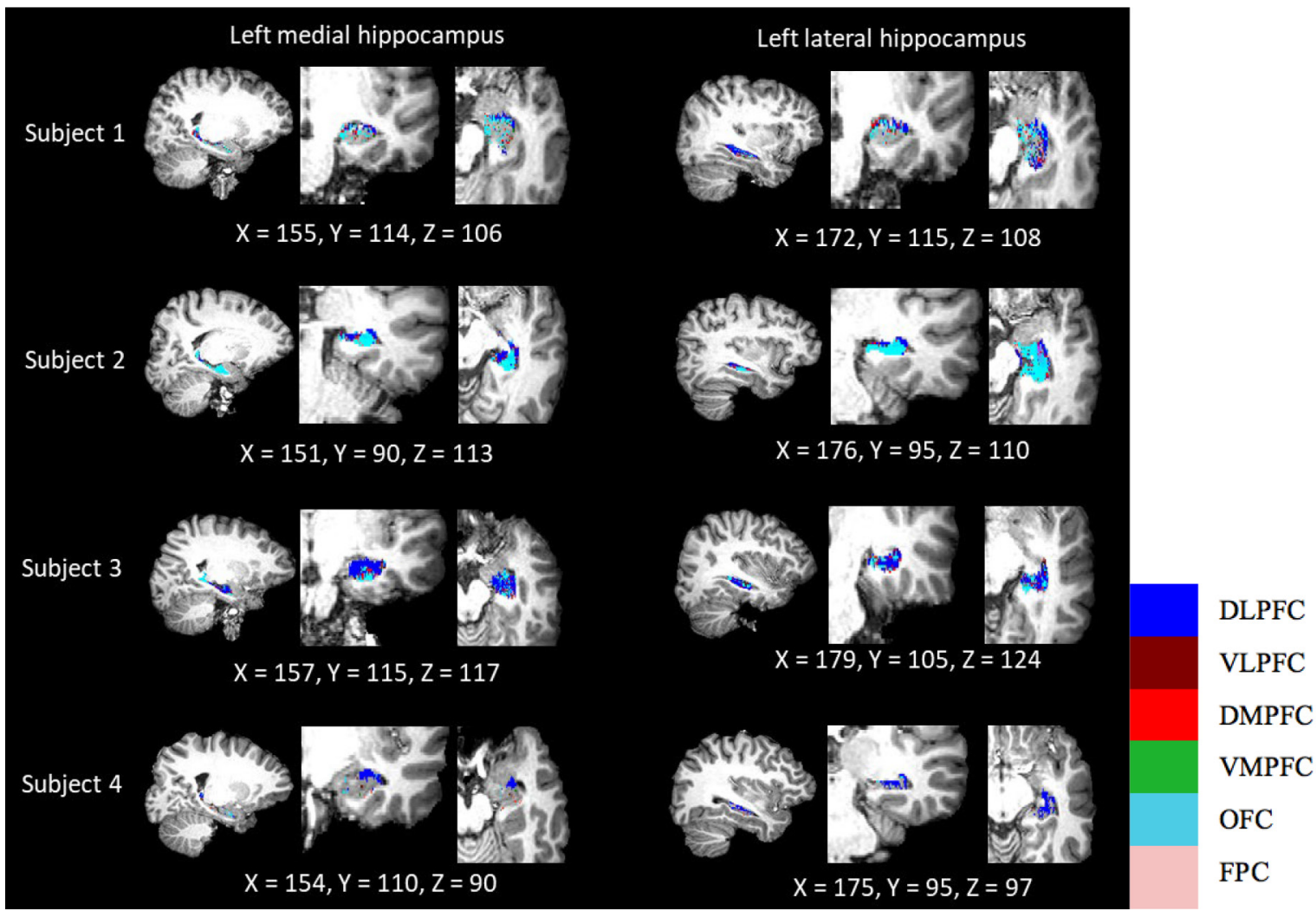

Figure 4. Parcellation of the hippocampus according to its connection probability to six PFC subdivisions in the left hemisphere $(n=4)$ 


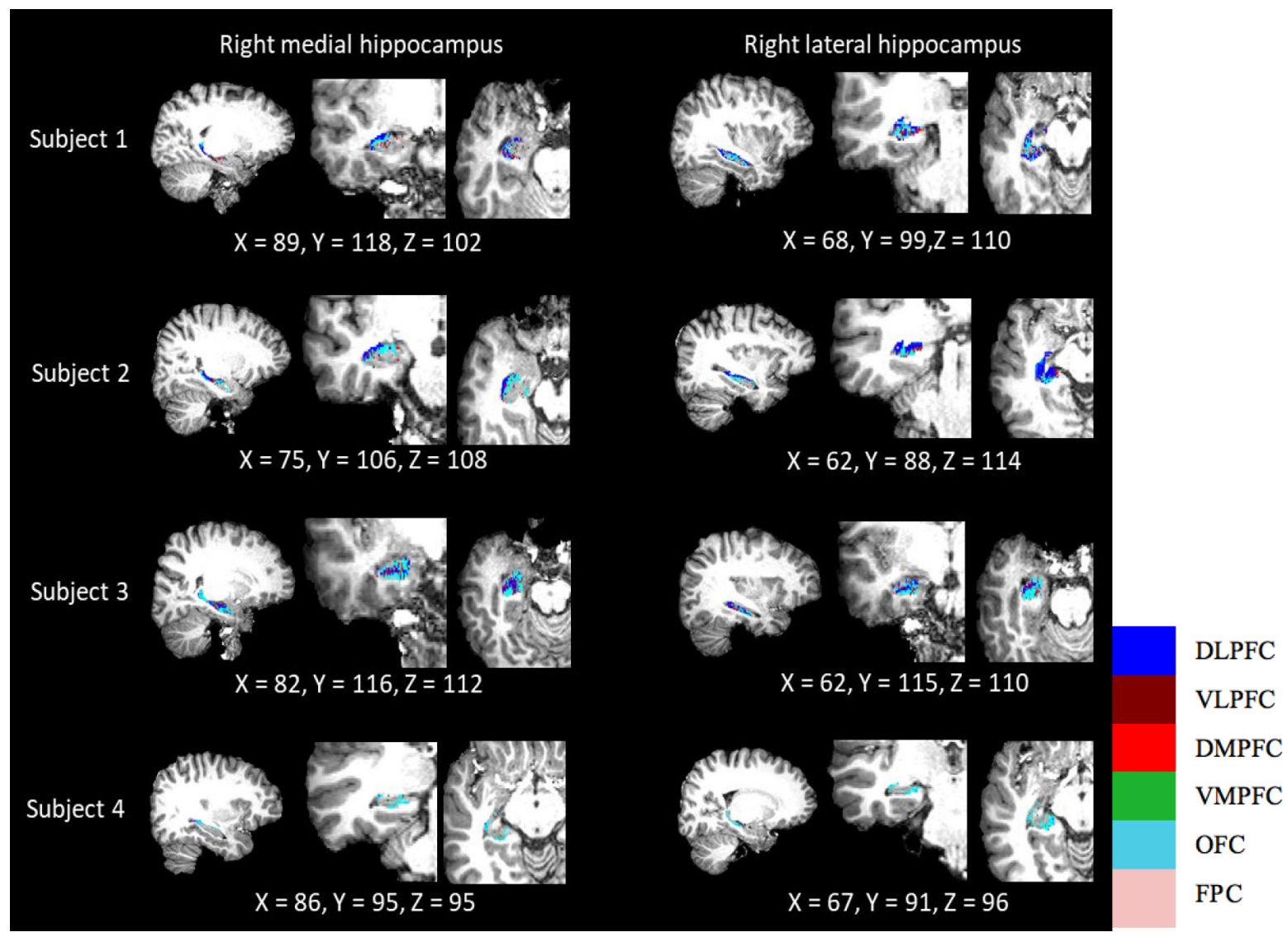

Figure 5. Parcellation of the hippocampus according to its connection probability to six PFC subdivisions in the right hemisphere $(n=4)$

ventral hippocampal subregions. Other regions with lower connection probabilities were barely seen in the parcellated map.

\section{Discussion}

Hippocampal-PFC connectivity consists of the main anatomical connection from the hippocampal formation to the PFC, either by monosynaptic or polysynaptic projections, which suggests a crucial role for the hippocampal-PFC circuit in the anatomical and functional coupling of the two regions (31). In the present study, we tracked hippocampal-PFC neural connectivity in four normal healthy human brains using probabilistic tractography.

Based on our findings, the connectivity of the hippocampus was mainly to the OFC of both hemispheres except for subject 4 (left hemisphere). For subject 4, the connectivity of the hippocampus in the left hemisphere was aberrated to the DLPFC region. However, we could not explain the aberration as the present study was conducted in a small sample $(n=4)$ and the analysis used a single-seed approach. A larger sample is preferred for the analysis of variability and ideally a network is constructed from the individual-based parcellation to better extract the variability from true subject difference.

The strong connectivity to the OFC may be explained by the similar functional roles of hippocampal formation and the OFC (32). Historically the hippocampus has been associated with mapping (33) and later, its role in encoding information about the world in a way that facilitates flexible and inferential cognitive processes has been put forward $(1,2,34-37)$. The OFC, by comparison, has traditionally been related to reward- and value-based behaviours $(38-45)$. However, it has recently been suggested that the OFC may have a cognitive-map-like function. Thus, it has been proposed that a fundamental function of the $\mathrm{OFC}$ is to form and to maintain neural representations of task state, that is, a representation of all the relevant internal and external stimuli or features that define a particular situation in the world $(46,47)$. 
Original Article | Parcellation of hippocampus

Because this function requires the $\mathrm{OFC}$ to encode both features of the environment (including observable sensory properties and unobservable, implicit variables that must be inferred) and how relationships between those features might change in different situations, the OFC has been described as a cognitive map of task state (47). From this view point, the OFC and hippocampus each contribute to cognitive mapping and the resultant behaviour (32).

Connectivity-based parcellation of the hippocampus according to its probabilistic connectivity to the PFC subdivisions in the four subjects studied were highly variable and produced no unifying pattern among them and did not conform to any established structural subregions of the hippocampus (48). This may suggest that the segmentation of the hippocampus does not follow its structural connectivity to PFC subdivisions. However, the small sample used does not make this conclusive. A recent study performed the functional parcellation of the hippocampus into head, body and tail parcels and found that functional parcellation did not strictly follow structural parcellation (49).

Several limitations of this study should be considered. First, as mentioned earlier, a larger sample size is required to confirm the present findings. Second, the fact that the hippocampus is composed of several regions, future studies should be conducted to find the connectivity from specific regions of the hippocampus to each PFC subdivision. Third, the use of probabilistic tractography can result in false positive and negative findings due to fibre complexity or partial volume effects throughout the white matter of the brain $(54,55)$. Fourth, tractography assumes monosynaptic connectivity and provides little or no information on indirect connectivity. Therefore, our results is to be read with caution.

\section{Conclusion}

In general, our preliminary findings indicate that in normal healthy subjects, the hippocampus showed the highest connectivity to the OFC as compared to other PFC subdivisions. We did not find a unifying pattern of distribution based on the connectivity-based parcellation of the hippocampus nor any indication that the connectivity follows structural subregions.

\section{Acknowledgements}

The authors would like to thank the volunteers for their participation and support of this research.

\section{Ethics of Study}

The study protocol was approved by the Human Research Ethics Committee of Universiti Sains Malaysia (USMJEPeM/15110486).

\section{Conflict of Interest}

None.

\section{Funds}

This work was supported by the Ministry of Higher Education (MOHE) Malaysia for its Transdisciplinary Research Grant Scheme (TRGS) number 203/PELECT/6768002, School of Medical Sciences for its Tabung Insentif Pembangunan Pengajian Siswazah PPSP (TIPPS) and funded by the USM fellowship scheme of Institute of Postgraduate Studies, Universiti Sains Malaysia.

\section{Authors' Contributions}

Conception and design: AHA

Analysis and interpretation of the data: ANA, ST

Drafting of the article: RZ

Critical revision of the article for important

intellectual content: AHA

Final approval of the article: AHA

Provision of study materials or patients: JMA

Administrative, technical, or logistic support:

JMA

Collection and assembly of data: ANA

\section{Correspondence}

Dr Asma Hayati Ahmad

MBBS (Universiti Malaya), MSc (USM),

DPhil (Oxon)

Department of Physiology,

School of Medical Sciences,

Universiti Sains Malaysia,

16150 Kubang Kerian, Kelantan, Malaysia.

Tel: +609 7676163

Fax: +609 7653370

E-mail: asmakck@usm.my 


\section{References}

1. Buzsáki G, Moser E. Memory, navigation and theta rhythm in the hippocampal-entorhinal system. Nat Neurosci. 2013;16(2):130-138. https://doi.org/10.1038/nn.3304

2. Eichenbaum H, Cohen NJ. Can we reconcile the declarative memory and spatial navigation views on hippocampal function? Neuron. 2014;83(4):764-770. https://doi.org/10.1016/j. neuron.2014.07.032

3. Gruart A, Leal-Campanario R, López-Ramos JC, Delgado-García JM. Functional basis of associative learning and its relationships with long-term potentiation evoked in the involved neural circuits: Lessons from studies in behaving mammals. Neurobiol Learn Mem. 2015;124:318. https://doi.org/10.1016/j.nlm.2015.04.006

4. Moser EI, Witter MP, Moser MB. Entorhinal cortex. In: Shepherd GM, Grillner S, editors. Handbook of brain microcircuits. Oxford: Oxford University Press; 2010.

5. Sigurdsson T, Duvarci S. Hippocampalprefrontal interactions in cognition, behaviour and psychiatric disease. Front Syst Neurosci. 2015;9:190. https://doi.org/10.3389/fnsys.2015 .00190

6. Bannerman DM, Rawlins JN, McHugh SB, Deacon RM, Yee BK, Bast T, et al. Regional dissociations within the hippocampus memory and anxiety. Neurosci Biobehav Rev. 2004;28(3):273-283. https://doi.org/10.1016/j. neubiorev.2004.03.004

7. Fanselow MS, Dong HW. Are the dorsal and ventral hippocampus functionally distinct structures? Neuron. 2010;65(1):7-19. https://doi .org/10.1016/j.neuron.2009.11.031

8. Strange BA, Witter MP, Lein ES, Moser EI. Functional organization of the hippocampal longitudinal axis. Nat Rev Neurosci. 2014;15(10):655-669. https://doi.org/10.1038/ nrn3785

9. Fuster JM. The prefrontal cortex. $5^{\text {th }}$ ed. New York: Academic Press; 2015.
10. Koenigs M, Grafman J. The functional neuroanatomy of depression: distinct roles for ventromedial and dorsolateral prefrontal cortex. Behav Brain Res. 2009;201(2):239-243. https://doi.org/10.1016/j.bbr.2009.03.004

11. Barbas H, Blatt GJ. Topographically specific hippocampal projections target functionally distinct prefrontal areas in the rhesus monkey. Hippocampus. 1995;5(6):511-533. https://doi.org/10.1002/hipo.450050604

12. Condé F, Maire-Lepoivre E, Audinat E, Crépel F. Afferent connections of the medial frontal cortex of the rat. II. Cortical and subcortical afferents. J Comp Neurol. 1995;352(4):567-593. https://doi.org/10.1002/cne.903520407

13. Hoover WB, Vertes RP. Anatomical analysis of afferent projections to the medial prefrontal cortex in the rat. Brain Struct Funct. 2007;212(2):149-179. https://doi.org/10.1007/ s00429-007-0150-4

14. Rosene DL, Van Hoesen GW. Hippocampal efferents reach widespread areas of cerebral cortex and amygdala in the rhesus monkey. Science. 1977;198(4314):315-317. https://doi .org/10.1126/science.4101022

15. Cassel JC, Pereira de Vasconcelos A, Loureiro M, Cholvin T, Dalrymple-Alford JC, Vertes RP. The reuniens and rhomboid nuclei: neuroanatomy, electrophysiological characteristics and behavioral implications. Prog Neurobiol. 2013;111:34-52. https://doi.org/10.1016/j.pneurobio.2013.08.006

16. Vertes RP. Interactions among the medial prefrontal cortex, hippocampus and midline thalamus in emotional and cognitive processing in the rat. Neuroscience. 2006;142(1):1-20. https://doi.org/10.1016/j. neuroscience.2006.06.027

17. Behrens TE, Berg HJ, Jbabdi S, Rushworth MF, Woolrich MW. Probabilistic diffusion tractography with multiple fibre orientations: what can we gain? Neuroimage. 2007;34(1):144-155. https://doi.org/10.1016/j. neuroimage.2006.09.018

18. Smith SM, Jenkinson M, Woolrich MW, Beckmann CF, Behrens TE, Johansen-Berg H, et al. Advances in functional and structural MR image analysis and implementation as FSL. Neuroimage. 2004;23(Suppl 1):S208-S219. https://doi.org/10.1016/j.neuroimage.2004.07.051 
19. Parker GJ, Alexander DC. Probabilistic anatomical connectivity derived from the microscopic persistent angular structure of cerebral tissue. Philos Trans $R$ Soc Lond B Biol Sci. 2005;360(1457):893-902. https://doi.org/ 10.1098/rstb.2005.1639

20. Habas C, Cabanis EA. Cortical projections to the human red nucleus: a diffusion tensor tractography study with a 1.5 -T MRI machine. Neuroradiology. 2006;48(10):755-762. https:// doi.org/10.1007/s00234-006-0117-9

21. Jang SH, Kwon HG. Neural connectivity of the posterior body of the fornix in the human brain: diffusion tensor imaging study. Neurosci Lett. 2013;549:116-119. https://doi.org/10.1016/ j.neulet.2013.06.017

22. Kwon HG, Jang SH. Neural connectivity of the lateral geniculate body in the human brain: diffusion tensor imaging study. Neurosci Lett. 2014;578:66-70. https://doi.org/10.1016/ j.neulet.2014.06.033

23. Nucifora PG, Verma R, Lee SK, Melhem ER. Diffusion-tensor MR imaging and tractography: exploring brain microstructure and connectivity. Radiology. 2007;245(2):367-384. https://doi .org/10.1148/radiol.2452060445

24. Croxson PL, Johansen-Berg H, Behrens TE, Robson MD, Pinsk MA, Gross CG, et al. Quantitative investigation of connections of the prefrontal cortex in the human and macaque using probabilistic diffusion tractography. $J$ Neurosci. 2005;25(39):8854-8866. https:// doi.org/10.1523/JNEUROSCI.1311-05.2005

25. Duvernoy HM. The human brain: surface, blood supply, and three-dimensional sectional anatomy. New York: Springer-Verlag; 1999.

26. Ray $\mathrm{RD}$, Zald $\mathrm{DH}$. Anatomical insights into the interaction of emotion and cognition in the prefrontal cortex. Neurosci Biobehav Rev. 2011;36(1):479-501. https://doi.org/10.1016/j. neurobiorev.2011.08.005

27. Ahmad A. The role of the prefrontal cortex in pain modulation [PhD diss.]. Oxford University, UK; 2012.
28. Behrens TE, Woolrich MW, Jenkinson M, Johansen-Berg H, Nunes RG, Clare S, et al. Characterization and propagation of uncertainty in diffusion-weighted MR imaging. Magn Reson Med. 2003;50(5):1077-1088. https://doi.org/10 $.1002 / \mathrm{mrm} .10609$

29. Ciccarelli O, Behrens TE, Altmann DR, Orrell RW, Howard RS, Johansen-Berg H, et al. Probabilistic diffusion tractography: a potential tool to assess the rate of disease progression in amyotrophic lateral sclerosis. Brain. 2006;129(7):1859-1871. https://doi.org/10.1093/brain/awl10o

30. Samsir S, Zakaria R, Razak SA, Ismail MS, Rahim MZA, Lin CS, et al. Characterisation of the corticospinal tract using diffusion magnetic resonance imaging in unilateral and bilateral cerebral palsy patients. Malays $J$ Med Sci. 2018;25:68-78. https://doi.org/10.21315/ mjms2018.25.5.7

31. Li M, Long C, Yang L. Hippocampal-prefrontal circuit and disrupted functional connectivity in psychiatric and neurodegenerative disorders. Biomed Res Int. 2015;2015:810548. https://doi.org/10.1155/2015/810548

32. Wikenheiser AM, Schoenbaum G. Over the river, through the woods: cognitive maps in the hippocampus and orbitofrontal cortex. Nat Rev Neurosci. 2016;17(8):513-523. https://doi.org/ $10.1038 / \mathrm{nrn} .2016 .56$

33. O'Keefe J, Nadel L. The hippocampus as a cognitive map. Oxford: The Clarendon Press; 1978.

34. Dudchenko PA, Wood ER. Place fields and the cognitive map. Hippocampus. 2015;25(6):709712. https://doi.org/10.1002/hipo.22450

35. Eichenbaum H, Dudchenko P, Wood E, Shapiro M, Tanila H. The hippocampus, memory, and place cells: is it spatial memory or a memory space?. Neuron. 1999;23(2):209-226. https://doi.org/10.1016/so896-6273(oo)80773-4

36. Redish AD. Beyond the cognitive map: from place cells to episodic memory. Cambridge (MA): MIT Press; 1999.

37. Wikenheiser AM, Redish AD. Decoding the cognitive map: ensemble hippocampal sequences and decision-making. Curr Opin Neurobiol. 2015;32:8-15. https://doi.org/10.1016/j.conb .2014 .10 .002 
38. Gallagher M, McMahan RW, Schoenbaum G. Orbitofrontal cortex and representation of incentive value in associative learning. $J$ Neurosci. 1999;19(15):6610-6614. https://doi .org/10.1523/JNEUROSCI.19-15-06610.1999

39. Gottfried JA, O'Doherty J, Dolan RJ. Encoding predictive reward value in human amygdala and orbitofrontal cortex. Science. 2003;301(5636):1104-1107. https://doi.org/10 $.1126 /$ science.1087919

40. McDannald MA, Jones JL, Takahashi YK, Schoenbaum G. Learning theory: a driving force in understanding orbitofrontal function. Neurobiol Learn Mem. 2014;108:22-27. https://doi.org/10.1016/j.nlm.2013.06.003

41. O’Doherty J, Kringelbach ML, Rolls ET, Hornak J, Andrews C. Abstract reward and punishment representations in the human orbitofrontal cortex. Nat Neurosci. 2001;4(1):95-102. https://doi .org/10.1038/82959

42. Padoa-Schioppa C, Assad JA. Neurons in the orbitofrontal cortex encode economic value. Nature. 2006;441(7090):223-226. https://doi .org/10.1038/nature04676

43. Wallis JD. Orbitofrontal cortex and its contribution to decision-making. Annu Rev Neurosci. 2007;30:31-56. https://doi.org/10 $.1146 /$ annurev.neuro.30.051606.094334

44. Thorpe SJ, Rolls ET, Maddison S. The orbitofrontal cortex: neuronal activity in the behaving monkey. Exp Brain Res. 1983;49(1):93-115. https://doi.org/10.1007/ BFoo235545
45. Tremblay L, Schultz W. Relative reward preference in primate orbitofrontal cortex. Nature. 1999;398(6729):704-708. https://doi .org/10.1038/19525

46. Takahashi YK, Roesch MR, Wilson RC, Toreson K, O’Donnell P, Niv Y, et al. Expectancy-related changes in firing of dopamine neurons depend on orbitofrontal cortex. Nat Neurosci. 2011;14:15901597. https://doi.org/10.1038/nn.2957

47. Wilson RC, Takahashi YK, Schoenbaum G, Niv Y. Orbitofrontal cortex as a cognitive map of task space. Neuron. 2014;81(2):267-279. https://doi.org/10.1016/j.neuron.2013.11.005

48. Lerma-Usabiaga G, Iglesias JE, Insausti R, Greve DN, Paz-Alonso PM. Automated segmentation of the human hippocampus along its longitudinal axis. Hum Brain Mapp. 2016;37:3353-3367. https://doi.org/10.1002/hbm.23245

49. Cheng H, Zhu H, Zheng Q, Liu J, He G. Functional parcellation of the hippocampus by semi-supervised clustering of resting state fMRI data. Sci Rep. 2020;10:16402. https://doi .org/10.1038/s41598-020-73328-1

50. Wedeen VJ, Wang RP, Schmahmann JD, Benner T, Tseng WY, Dai G, et al. Diffusion spectrum magnetic resonance imaging (DSI) tractography of crossing fibers. Neuroimage. 2008;41(4):1267-1277. https://doi.org/10.1016/ j.neuroimage.2008.03.036

51. Yamada K, Sakai K, Akazawa K, Yuen S, Nishimura T. MR tractography: a review of its clinical applications. Magn Reson Med Sci. 2009;8(4):165-174. https://doi.org/10.2463/ mrms.8.165 